\title{
Injectable meshes for neural recordings
}

Injection of a flexible mesh containing electronics enables neural recordings in the mouse brain in a minimally invasive fashion.

Neural activity is often recorded with rigid electrode arrays that are implanted via surgical openings in the skull. The inflexible nature of such electrodes can require compromises in their positioning and may lead to shifts in their location during long-term recordings. To overcome these issues, ideal recording electrodes would be flexible and integrate into the tissue. Charles Lieber and his team at Harvard University have developed flexible meshes that can be injected into biomaterials and tissues and that contain electronics suitable for recording neural activity or other applications.

According to Lieber, the idea of flexible, injectable electronics has evolved over several years and builds

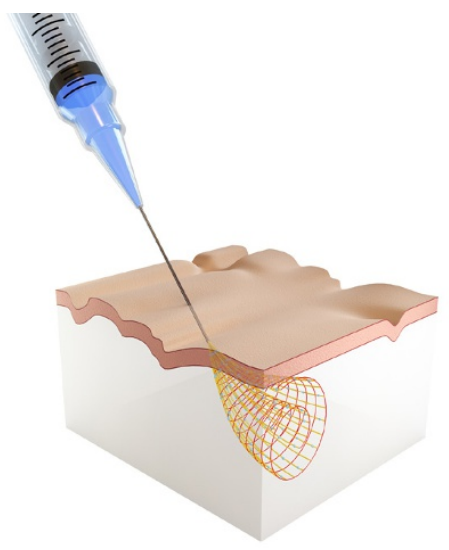

Injecting electronics with a syringe. Reproduced from Liu. et al., Nature Publishing Group. on tissue engineering work in his lab. He had become increasingly disillusioned with chip-based work and was looking for less invasive ways to interface with tissues. This required "reducing overall feature sizes, increasing flexibility and making the connectivity look more and more like something that is natural," says Lieber.

In addition to the flexibility of the material, the ability to simply inject the electronics further reduces the invasiveness of the tool. However, to allow the meshes to be inserted into syringes,

\section{CHEMICAL BIOLOGY}

\section{EFFICIENT GENERATION OF PROTEINS WITH SITE- SPECIFIC PHOSPHOSERINES}

\section{An optimized strategy allows genetic encoding of phosphoserine and its nonhydrolyzable analog.}

Phosphorylation is an important post-translational modification that can change a protein's function by altering features such as localization, activity or binding interactions. However, the effects of phosphorylation can be difficult to study, as methods for generating or isolating proteins that are phosphorylated at a specific location are challenging.

Jason Chin at the Medical Research Council Laboratory of Molecular Biology explains that for phosphorylation, "even though you might know that a specific modification occurs in a cell in a natural protein, there is often no way to make that modified protein because in many cases the enzyme that makes the modification is unknown." He adds that even for cases where the specific phosphorylating enzyme, or kinase, is known, it can be difficult to phosphorylate a single, specific site to completion.

These challenges have important consequences for our understanding of protein function. "In many cases we have very little idea what these modifications do," says Chin. The team, including Daniel Rogerson, a graduate student in Chin's laboratory, began working on a potential solution: a special tRNA and aminoacyl-tRNA synthetase (SepRS) pair from methanogenic archaea in which the tRNA is aminoacylated by the synthetase with phosphoserine (pSer). This pair, in principle, can be used to encode pSer into proteins.

To label proteins in specific locations, the researchers first mutated the tRNA anticodon to recognize a specific stop codon, allowing the pSer to be incorporated where that stop codon has been introduced into a gene. However, mutating the anticodon made the addition of pSer to the tRNA by SepRS much less efficient. "The aminoacyl synthetase recognizes the natural anticodon of the tRNA very strongly, so when you change the 
the researchers had to make some simple adjustments to the mesh design. "You don't want this to crumple up like a ball of aluminum foil or it will get jammed in the needle. You want it to scroll up like a piece of paper ... inside the needle," says Lieber. They achieved this by giving the mesh subunits a parallelogram shape, which reduced stiffness in the direction that the mesh rolls up while maintaining rigidity in the other direction.

Once the mesh is injected, its behavior depends on the surrounding tissue or material. Within soft, flexible surroundings, the mesh can expand and unfold. However, when injected into the dense tissue of a mouse brain, it can expand only a little and thus does not cut into and damage the tissue. More importantly, the mesh has mechanical properties and feature sizes similar to those of neural tissue, yielding almost no immunoreactivity in contrast to other electrodes.

The researchers demonstrated the suitability of the injectable electronics by using them to record local field potentials in the mouse hippocampus. Standard analysis methods even allowed them to identify single-unit action potentials in their recordings. A benefit of the injectable electronics is that the researchers can position them with a precision of 10 micrometers, and they know exactly where the recording electrodes are located on the mesh. Lieber thinks that these meshes can even be placed more precisely than rigid probes. In addition, the injected meshes enable chronic recordings, as they are incorporated into the tissue and their position remains stable. "We are able to record really nice single-unit data over literally 4 months," mentions Lieber.

Finally, the injectable electronics enable experiments that were not previously possible. In collaboration with Josh Sanes at Harvard University, Lieber has started to perform electrophysiological recordings in the mouse retina, which was previously not accessible in vivo. Because the injectable electronics are essentially transparent, they do not interfere with vision. Lieber even dreams of recording from multiple sites simultaneously, such as the retina and the visual cortex, which should be possible as the electronics are so easy to inject.

Nina Vogt

RESEARCH PAPERS

Liu, J. et al. Syringe-injectable electronics. Nat. Nanotechnol. 10, 629-636 (2015).

anticodon ... that basically destroys the recognition between the synthetase and the tRNA," says Chin.

Structural and biochemical information suggested that changing nucleotides adjacent to the anticodon region might compensate for the anticodon mutations. So the team generated a mutant library of tRNAs and screened for the most efficiently aminoacylated ones. After identifying winners, they also screened SepRS mutants to identify those that would allow enhanced aminoacylation of the optimized tRNA. From these experiments, the researchers were able to find mutant tRNA-SepRS combinations that were roughly 20 times more efficient than other systems that used this pair for pSer incorporation.

The team was able to generate several phosphorylated proteins using their optimized system, including myoglobin, ubiquitin and the kinase Nek7, and found that pSer incorporation was highly efficient. They were able to generate large quantities of specifically labeled, homogeneous proteins, which could then be used in follow-up biochemical assays.

Chin recalls that one of the most surprising aspects of the work was that the system could be developed to incorporate nonhydrolyzable pSer analogs into proteins. To achieve this, the team mutated the Escherichia coli genome to deplete the available pSer in the cell. They found that the nonhydrolyzable analogs they added to cells were efficiently incorporated into proteins using these genetically modified strains. Again, they were able to achieve high yields of specifically labeled proteins.

These developments have important ramifications for biochemistry and structural biology, allowing researchers to generate large amounts of homogeneously phosphorylated proteins for biochemical assays and structural studies such as X-ray crystallography for the first time. Chin also hopes to extend the work to genetically encode other phosphorylated amino acids such as phosphothreonine.

\section{Rita Strack}

RESEARCH PAPERS

Rogerson, D.T. et al. Efficient genetic encoding of phosphoserine and its nonhydrolyzable analog. Nat. Chem. Biol. 11, 496-503 (2015). 